\title{
Nonlinear Wave Modulation in Nanorods Based on Nonlocal Elasticity Theory by Using Multiple-Scale Formalism
}

\author{
Guler Gaygusuzoglu \\ Namik Kemal University, Corlu Faculty of Engineering, Department of Civil Engineering, \\ Corlu-Tekirdag. \\ E-mail address: ggaygusuzoglu@nku.edu.tr
}

Received date: 11.05 .2018

Accepted date: 26.09 .2018

\begin{abstract}
Many systems in physics, engineering, and natural sciences are nonlinear and modeled with nonlinear equations. Wave propagation, as a branch of nonlinear science, is one of the most widely studied subjects in recent years. Nonlocal elasticity theory represents a common growing technique used for conducting the mechanical analysis of microelectromechanical and nanoelectromechanical systems. In this study, nonlinear wave modulation in nanorods was examined by means of nonlocal elasticity theory. The nonlocal constitutive equations of Eringen were utilized in the formulation, and the nonlinear equation of motion of nanorods was obtained. By applying the multiple scale formalism, the propagation of weakly nonlinear and strongly dispersive waves was investigated, and the Nonlinear Schrödinger (NLS) equation was obtained as the evolution equation. A part of spacial solutions of the NLS equation, i.e. nonlinear plane wave, solitary wave and phase jump solutions, were presented. In order to investigate the nonlocal impacts on the NLS equation numerically, whether envelope solitary wave solutions exist was investigated by utilizing the physical and geometric features of carbon nanotubes (CNTs).
\end{abstract}

Keywords: Nanorods, nonlinear wave modulation, nonlocal elasticity theory, multiple-scale method.

\section{Introduction}

The accurate characterization of the actual mechanical behavior of nanoscale devices is significant in the design of the devices in question, including CNTs. However, the application of a classical continuum theory is questionable while carrying out the mechanical analysis of carbon nanotubes. The classical continuum theory (classical elasticity theory) is length scalefree. Hence, it cannot accurately account for very small-sized effects. To eliminate the deficiencies of the classical continuum theory, different higher-order continuum theories, such as micro-polar elasticity theory [1-4], nonlocal elasticity theory [5-7], couple stress theory [8] and the modified couple stress approach [9, 10], have received significant attention in the analysis of micro- and nanostructures. Due to the high cost of experiments that operate on the nanoscale, it is of vital importance to introduce suitable physical models for nanobeams (carbon nanotubes) for the establishment of an appropriate theoretical and mathematical framework for nanosized structures [11-13]. Eringen [14] and Eringen and Edelen [15] proposed nonlocal elasticity theory in the 1970s for the purpose of overcoming the deficiencies of classical 
elasticity models. Unlike the conventional theory of elasticity, in the nonlocal theory of elasticity, it is assumed that the strain at a particular point in a continuous domain and the strain at all points in the domain determine the stress at the point in question. Several studies have been performed using this nonlocal model to conduct the analysis of the mechanical behavior of nanosized structures [16-18].

Wave propagation is a very effective, nondestructive method used for the characterization of nanostructures. Nanosensor transducers also work on the wave propagation principle. The wave propagation issue has attracted attention around the world [19-25] in various domains of science and engineering due to its importance. In the study carried out by Lim and Yang [19], the researchers investigated wave propagation in CNTs based on nonlocal elastic stress field theory and Timoshenko beam theory and acquired a novel dispersion and spectrum correlation. In the study of $\mathrm{Hu}$ et al. [20], transverse and torsional waves in single-walled carbon nanotubes (SWCNTs) and double-walled carbon nanotubes (DWCNTs) were investigated on the basis of nonlocal elastic cylindrical shell theory. The researchers compared the wave dispersion that was estimated by utilizing their model with molecular dynamics simulations in the terahertz area and concluded that it was possible to acquire a better prediction of dispersion relations by the nonlocal model. Wu and Dzenis [26] investigated wave propagation in nanofibers. The researchers studied longitudinal and flexural wave propagation in nanofibers by employing local theories in terms of surface impacts. Challamel [27] suggested a dispersive wave equation by utilizing nonlocal elasticity. The researcher introduced a mixture theory of a local and nonlocal strain. Narendar and Gopalakrishnan [28] investigated the nonlocal scale impacts on the ultrasonic wave feature of nanorods by employing the nonlocal Love rod theory. In the study of Narendar [29], the nonlocal Love-Rayleigh rod theory was used to examine wave propagation in uniform nanorods.

It is well-known that in the case of a sufficiently small amplitude of a wave, a lot of nonlinear systems allow for harmonic wave solutions with sufficiently small nonlinear terms for ignoring them, and the amplitude stays unchanged over time. In case of a small-but-finite amplitude of the wave, it is not possible to ignore the nonlinear terms, which causes an alteration in amplitude in space as well as time. In case of the slow variation of the amplitude during the period of oscillation, a stretching transformation allows the separation of the system into a quickly changing component related to the oscillation and a slowly changing component, for example, the amplitude. It is possible to present a formal solution as an asymptotic expansion, and it is possible to derive an equation that identifies the modulation of the first order amplitude. For example, the nonlinear Schrödinger (NLS) equation represents the most elementary representative equation that defines the self-modulation of one-dimensional monochromatic plane waves in dispersive media. The equilibrium between dispersion and nonlinearity is presented by it. The problem of nonlinear wave modulation was studied by Erbay, Erbay, and Dost [30] in micropolar elastic media longitudinal waves. They showed that the nonlinear Schrödinger (NLS) equation, originating from the equilibrium between nonlinearity and dispersion, governs the nonlinear self-modulation of a longitudinal microrotation wave in micropolar elastic media. Erbay and Erbay [31] studied nonlinear self-modulation in distensible tubes filled with fluid by utilizing the nonlinear equations of a thin viscoelastic tube and the approximate fluid equations. As a result of the study, the researchers indicated that the dissipative NLS equation governs the nonlinear modulation of pressure waves. The amplitude modulation of the fluid-filled viscoelastic tube was investigated in the study of Akgun and Demiray [32], and the dissipative NLS equation was acquired. Furthermore, Akgun and Demiray [33] studied the modulation of non-linear axial and transverse waves in a thin elastic 
tube filled with fluid and obtained nonlinear Schrödinger equation which corresponds to two nonlinear equations related to the axial and transverse motions of the tube material. Erbay, Erbay, and Erkip [34] studied a unidirectional wave motion in a nonlocally and nonlinearly elastic medium. Duruk, Erbay, and Erkip [35] investigated the blow-up and global existence for a general class of nonlocal nonlinear coupled wave equations. In the previous studies, nonlinear wave modulation in nanotubes has not been considered.

In this study, the amplitude modulation of nonlinear wave propagation in nanorods based on the nonlocal theory was studied by employing the reductive perturbation technique. Firstly, a one-dimensional nonlinear field equation was obtained. The linear dispersion relation of axial waves was also presented for observing the dispersive characteristic of the medium. It is shown that the nonlinear Schrödinger (NLS) equation governs the nonlinear self-modulation of axial waves in nonlocal elastic media and it is given as an analytical plane wave, envelope solitary wave and a phase jump solution for the NLS equation. For the purpose of numerical investigation of the nonlocal impacts on the NLS equation, whether envelope solitary wave solutions exist was investigated by utilizing the physical and geometric features of carbon nanotubes.

The organization of the current study is presented below. Section 2 contains information on nonlocal elasticity theory and the governing equation of the system. The basics of the method of multiple scale formalism and self-modulation of nonlinear waves are discussed in Section 3. Section 4 contains the numerical and graphical presentation of the findings. In Section 5, some discussions and conclusions are given.

\section{Basic equations and theoretical preliminaries}

In the present section, the basic equation that governs the motion of nanorods in nonlocal elastic media should be derived. The constitutive equation of the nonlocal linear vibration of a nanorod which is provided by Eringen [15], Aydogdu [17] is as follows;

$$
\left[1-\left(\mathrm{e}_{0} a\right)^{2} \frac{\partial}{\partial \mathrm{x}^{2}}\right] \tau_{k l}=\lambda_{L} \varepsilon_{\mathrm{rr}} \delta_{k l}+2 \mu_{L} \varepsilon_{k l}
$$

where $\tau_{k l}$ represents the nonlocal stress tensor, $\varepsilon_{k l}$ represents the strain tensor, $\lambda_{L}$ and $\mu_{L}$ represent Lame constants, $a$ represents the internal characteristic length, and $\mathrm{e}_{0}$ represents a constant. From now on, the nonlocal parameter $\mu$ will be used as $\left(\mathrm{e}_{0} a\right)^{2}=\mu$.

Selection of the $e_{0}$ parameter (in a unit of length) is very important in ensuring the accuracy of nonlocal models. It is possible to write Eq.(1) for one-dimensional rod case as follows;

$$
\left[1-\mu \frac{\partial}{\partial \mathrm{x}^{2}}\right] \tau_{\mathrm{kl}}=\mathrm{E}_{\mathrm{E}} \varepsilon_{k l}
$$


where $\mathrm{E}_{\mathrm{E}}$ represents the modulus of elasticity. It is possible to write the equation of motion for a rod with axial vibration motion as follows;

$$
\frac{\partial \mathrm{N}^{\mathrm{L}}}{\partial \mathrm{x}}=\mathrm{m} \frac{\partial^{2} \mathrm{u}(\mathrm{x}, \mathrm{t})}{\partial \mathrm{t}^{2}}
$$

where $\mathrm{u}(\mathrm{x}, \mathrm{t})$ denotes the axial displacement, and m gives the mass per unit length, while $\mathrm{N}^{\mathrm{L}}$ provides the axial force per unit length for local elasticity and is presented as follows;

$$
\mathrm{N}^{\mathrm{L}}=\int_{\mathrm{A}} \sigma_{\mathrm{xx}} \mathrm{dA}
$$

where A represents the cross-sectional area, $\sigma_{\mathrm{xx}}$ represents the local stress component in the xdirection. By taking the area integral of equation (2), the following equation can be obtained;

$$
\mathrm{N}-\mu \frac{\partial^{2}}{\partial \mathrm{x}^{2}} \mathrm{~N}=\mathrm{N}^{\mathrm{L}}
$$

Here $\mathrm{N}=\int_{\mathrm{A}} \sigma_{\mathrm{xx}} \mathrm{dA}$ indicates axial force per unit length in nonlocal elasticity. The axial vibration equation of the rod in nonlocal elasticity may be reached using equations (3) and (5) in terms of the displacement component as follows;

$$
E_{E} A \frac{\partial^{2} u}{\partial x^{2}}=\left[1-\mu \frac{\partial}{\partial x^{2}}\right] m \frac{\partial^{2} u(x, t)}{\partial t^{2}}
$$

The equation above represents the basic equation of the nonlocal rod model for axial vibration in a thin rod. In case of $\mu=\mathrm{e}_{0} a=0$, the reduction of the equation to the equation of the classical rod model is performed. To obtain the nonlinear vibration equation of the nanotube in an elastic medium, first we introduce the deformation gradient tensor that was described by Malvern [36] as follows;

$$
\mathrm{F}=\vec{\nabla} \mathrm{u}+\mathrm{I}
$$

Here, $\mathrm{u}$ represents the displacement component of the motion, while I is the unit matrix. If body forces on the element are absent in a medium exposed to a finite extension, in terms of material coordinates, it is possible to write the equations of motion as follows;

$$
\nabla \cdot\left[\mathrm{SF}^{\mathrm{T}}\right]=\rho_{0} \frac{\partial^{2} \mathrm{u}}{\partial \mathrm{t}^{2}}
$$

Here $\rho_{0}$ is the non-deformed density of the medium, while $S$ is the second Piola-Kirchoff stress tensor. The second Piola-Kirchoff stress tensor represents a conjugate of the Green strain tensor 
in terms of energy. Therefore, Hook's law can be used as the governing equation. The equation is written as follows;

$$
\mathrm{S}=\mathrm{cE}
$$

Here, $\mathrm{c}$ denotes the fourth-order tensor that represents the elastic behavior of the material, while E denotes the Green strain tensor written as follows;

$$
\mathrm{E}=\frac{1}{2}\left[\mathrm{~F}^{\mathrm{T}} \mathrm{F}-1\right]
$$

If limiting the boundary conditions of the rod and assuming only the radial deformation $U(x, t)$ take place in the medium, the gradient deformation tensor in the cartesian coordinates becomes a diagonal matrix.

$$
\begin{gathered}
\mathrm{F}_{\mathrm{xx}}=1+\frac{\partial \mathrm{U}}{\partial \mathrm{x}} \\
\mathrm{F}_{\mathrm{zz}}=1 \\
\mathrm{~F}_{\mathrm{yy}}=1
\end{gathered}
$$

By referring only to the non-zero element in the Green strain tensor, the following equation can be obtained:

$$
\mathrm{E}_{\mathrm{xx}}=\left(1+\frac{1}{2} \frac{\partial \mathrm{U}}{\partial \mathrm{x}}\right) \frac{\partial \mathrm{U}}{\partial \mathrm{x}}
$$

The stress-strain relations of isotropic materials with the modulus of elasticity E $\mathrm{E}_{\mathrm{E}}$ and Poisson's ratio $v$ become as follows;

$$
\mathrm{S}_{\mathrm{ij}}=\frac{E_{E}}{(1+v)}\left[\mathrm{E}_{\mathrm{ij}}+\frac{v}{1-2 v} \mathrm{E}_{\mathrm{kk}} \delta_{\mathrm{ij}}\right]
$$

$\delta_{\mathrm{ij}}$ denotes the Kronecker delta. If we replace equation (14) into equation (15), it is observed that shear stresses are eliminated, while normal stress elements are shown as below:

$$
\begin{gathered}
\mathrm{S}_{\mathrm{xx}}=\frac{E_{E}(1-v)}{(1+v)(1-2 v)}\left(1+\frac{1}{2} \frac{\partial \mathrm{U}}{\partial \mathrm{x}}\right) \frac{\partial \mathrm{U}}{\partial \mathrm{x}} \\
\mathrm{S}_{\mathrm{yy}}=\mathrm{S}_{\mathrm{zz}}=\frac{E_{E} v}{(1+v)(1-2 v)}\left(1+\frac{1}{2} \frac{\partial \mathrm{U}}{\partial \mathrm{x}}\right) \frac{\partial \mathrm{U}}{\partial \mathrm{x}}
\end{gathered}
$$


Equation (8) can be obtained as follows by rearranging equations (16), (17) and using equations (11), (12), (13):

$$
\left[\left(\frac{\partial U}{\partial x}\right)^{2}+2 \frac{\partial U}{\partial x}+\frac{2}{3}\right] \frac{\partial^{2} U}{\partial x^{2}}=\frac{2 \rho_{0}(1+v)(1-2 v)}{3 E_{E}(1-v)} \frac{\partial^{2} U}{\partial t^{2}}
$$

In a special case, the infinite deformation of the medium, the nonlinear terms in equation (18) become insignificant, and equation (18) is reduced to the following equation.

$$
\frac{\partial^{2} \mathrm{U}}{\partial \mathrm{x}^{2}}=\frac{\rho_{0}(1+v)(1-2 v)}{E_{E}(1-v)} \frac{\partial^{2} \mathrm{U}}{\partial \mathrm{t}^{2}}
$$

By making the equation non-dimensional, let us introduce the following non-dimensional variables:

$$
\begin{gathered}
\Psi=\frac{\mathrm{U}}{r_{0}} \\
\zeta=\frac{\mathrm{x}}{r_{0}}
\end{gathered}
$$

here $r_{0}$ defines the radius of the rod. If equations (18) and (19) are reorganized by using equations (21) and (22), the following non-dimensional equations can be obtained as has been described by Mousavi and Fariborz [37] ;

$$
\left[\left(\frac{\partial \psi}{\partial \zeta}\right)^{2}+2\left(\frac{\partial \psi}{\partial \zeta}\right)+\frac{2}{3}\right] \frac{\partial^{2} \psi}{\partial \zeta^{2}}=\frac{2}{3} \delta \frac{\partial^{2} \psi}{\partial t^{2}}
$$

and

$$
\frac{\partial^{2} \psi}{\partial \zeta^{2}}=\delta \frac{\partial^{2} \psi}{\partial \mathrm{t}^{2}}
$$

where the coefficient $\delta$ is defined as follows:

$$
\delta=\frac{\rho_{0} r_{0}^{2}(1+v)(1-2 v)}{E_{E}(1-v)} .
$$

By using Eqs. (2), (8), (16) and (17), the following nonlinear equation of motion in terms of nonlocal elasticity is obtained: 


$$
\left[\left(\frac{\partial \psi}{\partial \zeta}\right)^{2}+2\left(\frac{\partial \psi}{\partial \zeta}\right)+\frac{2}{3}\right] \frac{\partial^{2} \psi}{\partial \zeta^{2}}=\frac{2}{3} \delta \frac{\partial^{2} \psi}{\partial \mathrm{t}^{2}}-\frac{2}{3} \delta \mu \frac{\delta^{4} \psi}{\delta \zeta^{2} \delta \mathrm{t}^{2}}
$$

A similar equation was obtained by Fernandes et al. [38]. The main difference is that they neglected y and $\mathrm{z}$ component contributions.

Setting $\mu=0$ leads to the nonlinear equation of motion of classical elasticity.

\section{Nonlinear wave modulation in nanorods by using the multiple-scale method}

Finding precise solutions for nonlinear problems is usually hard. This especially applies to nonlinear dynamics under the nonlocal elasticity theory due to the apparent complexity of the governing equations. Nevertheless, handling nonlinear problems in case of sufficiently weak nonlinearity is relatively straightforward. In this situation, evolution equations originating from the equilibrium between dispersion and nonlinearity can be obtained using the dispersive nature of the medium. As a result of the above-mentioned characteristic, it is possible to apply the farfield theory of weakly nonlinear waves, the complete development of which has been performed in different areas of engineering and physics, to nonlocal elasticity theory in case of equilibrium between nonlinearity and dispersion.

In the present section, the modulation of the axial waves in nonlocal elastic media due to nonlinear effects is examined. Therefore, the multiple scale technique [39] is used, and the coordinate stretching below is presented:

$$
\zeta_{n}=\varepsilon^{n} \zeta \quad, \quad t_{n}=\varepsilon^{n} t \quad, \quad(n=0,1,2, \ldots)
$$

where $\varepsilon$ represents a small parameter measuring the weakness of non-linearity.

It should be assumed that the field quantities represent the functions of fast variables $(\zeta, t)$, as well as slow variables $\left(\zeta_{0}, \zeta_{1}, \zeta_{2}, \ldots ; t_{0}, t_{1}, t_{2}, \ldots\right)$. Therefore, the substitution presented below can be performed:

$$
\frac{\partial}{\partial \zeta}=\sum_{n=0}^{N} \varepsilon^{n} \frac{\partial}{\partial \zeta_{n}} \quad, \quad \frac{\partial}{\partial t}=\sum_{n=0}^{N} \varepsilon^{n} \frac{\partial}{\partial t_{n}}
$$

By performing the expansion of the field quantities into an asymptotic series of $\varepsilon$ as:

$$
\psi=\sum_{n=1}^{\infty} \varepsilon^{n} \Psi_{n}\left(\zeta_{0}, \zeta_{1}, \zeta_{2}, \ldots ; t_{0}, t_{1}, t_{2}, \ldots\right)=\varepsilon \psi_{1}+\varepsilon^{2} \Psi_{2}+\varepsilon^{3} \Psi_{3}+\ldots
$$


and introducing the above-mentioned expansion into Eq. (26), the differential equation presented below is acquired.

$$
\begin{aligned}
& {\left[\left(\varepsilon \frac{\partial \Psi_{1}}{\partial \zeta_{0}}\right)^{2}+2 \varepsilon^{3} \frac{\partial \Psi_{1}}{\partial \zeta_{0}} \frac{\partial \Psi_{1}}{\partial \zeta_{1}}+2 \varepsilon \frac{\partial \Psi_{1}}{\partial \zeta_{0}}+2 \varepsilon^{2} \frac{\partial \Psi_{2}}{\partial \zeta_{0}}+2 \varepsilon^{2} \frac{\partial \Psi_{1}}{\partial \zeta_{1}}+\cdots \frac{2}{3}\right] \times\left[\varepsilon \frac{\partial^{2} \psi_{1}}{\partial \zeta_{0}^{2}}+\varepsilon^{2} \frac{\partial^{2} \Psi_{2}}{\partial \zeta_{0}{ }^{2}}+\right.} \\
& \left.\varepsilon^{3} \frac{\partial^{2} \psi_{3}}{\partial \zeta_{0}{ }^{2}}+2 \varepsilon^{2} \frac{\partial^{2} \psi_{1}}{\partial \zeta_{0} \partial \zeta_{1}}+2 \varepsilon^{3} \frac{\partial^{2} \psi_{2}}{\partial \zeta_{0} \partial \zeta_{1}}+2 \varepsilon^{3} \frac{\partial^{2} \psi_{1}}{\partial \zeta_{0} \partial \zeta_{2}}+\varepsilon^{3} \frac{\partial^{2} \psi_{1}}{\partial \zeta_{1}{ }^{2}}\right]=\frac{2 \delta}{3}\left[\varepsilon \frac{\partial^{2} \psi_{1}}{\partial t_{0}{ }^{2}}+\varepsilon^{2} \frac{\partial^{2} \psi_{2}}{\partial t_{0}{ }^{2}}+\varepsilon^{3} \frac{\partial^{2} \psi_{3}}{\partial t_{0}{ }^{2}}+\right. \\
& \left.2 \varepsilon^{2} \frac{\partial^{2} \psi_{1}}{\partial t_{0} \partial t_{1}}+2 \varepsilon^{3} \frac{\partial^{2} \psi_{2}}{\partial t_{0} \partial t_{1}}+2 \varepsilon^{3} \frac{\partial^{2} \psi_{1}}{\partial t_{0} \partial t_{2}}+\varepsilon^{3} \frac{\partial^{2} \psi_{1}}{\partial t_{1}{ }^{2}}\right]-\frac{2 \delta \mu}{3}\left[\varepsilon \frac{\partial^{4} \psi_{1}}{\partial t_{0}{ }^{2} \partial \zeta_{0}{ }^{2}}+\varepsilon^{2} \frac{\partial^{4} \Psi_{2}}{\partial t_{0}{ }^{2} \partial \zeta_{0}{ }^{2}}+\right. \\
& \varepsilon^{3} \frac{\partial^{4} \Psi_{3}}{\partial t_{0}{ }^{2} \partial \zeta_{0}{ }^{2}}+2 \varepsilon^{2} \frac{\partial^{4} \Psi_{1}}{\partial t_{0}{ }^{2} \partial \zeta_{0} \partial \zeta_{1}}+2 \varepsilon^{3} \frac{\partial^{4} \Psi_{2}}{\partial t_{0}{ }^{2} \partial \zeta_{0} \partial \zeta_{1}}+2 \varepsilon^{3} \frac{\partial^{4} \Psi_{1}}{\partial t_{0}{ }^{2} \partial \zeta_{0} \partial \zeta_{1}}+\varepsilon^{3} \frac{\partial^{4} \Psi_{1}}{\partial t_{0}{ }^{2} \partial \zeta_{1}{ }^{2}}+ \\
& \left.2 \varepsilon^{2} \frac{\partial^{4} \Psi_{1}}{\partial \zeta_{0}^{2} \partial t_{0} \partial t_{1}}+2 \varepsilon^{3} \frac{\partial^{4} \Psi_{2}}{\partial \zeta_{0}^{2} \partial t_{0} \partial t_{1}}+4 \varepsilon^{3} \frac{\partial^{4} \Psi_{1}}{\partial t_{0} \partial t_{1} \partial \zeta_{0} \partial \zeta_{1}}+2 \varepsilon^{3} \frac{\partial^{4} \Psi_{1}}{\partial \zeta_{0}^{2} \partial t_{0} \partial t_{2}}+\varepsilon^{3} \frac{\partial^{4} \Psi_{1}}{\partial t_{1}^{2} \partial \zeta_{0}^{2}}\right]
\end{aligned}
$$

The set of differential equations presented below is acquired as a result of setting the coefficients of like powers of $\varepsilon$ equal to zero;

First-order, $O(\varepsilon)$, equation:

$$
\frac{\partial^{2} \Psi_{1}}{\partial \zeta_{0}^{2}}=\delta \frac{\partial^{2} \Psi_{1}}{\partial \mathrm{t}_{0}{ }^{2}}-\delta \mu \frac{\partial^{4} \Psi_{1}}{\partial \mathrm{t}_{0}{ }^{2} \partial \zeta_{0}{ }^{2}}
$$

Second-order, $O\left(\varepsilon^{2}\right)$,equation:

$$
\begin{aligned}
& 2 \frac{\partial \Psi_{1}}{\partial \zeta_{0}} \frac{\partial^{2} \Psi_{1}}{\partial \zeta_{0}^{2}}+\frac{2}{3} \frac{\partial^{2} \Psi_{2}}{\partial \zeta_{0}^{2}}+\frac{4}{3} \frac{\partial^{2} \psi_{1}}{\partial \zeta_{0} \partial \zeta_{1}}=\frac{2 \delta}{3} \frac{\partial^{2} \Psi_{2}}{\partial t_{0}^{2}}+\frac{4 \delta}{3} \frac{\partial^{2} \Psi_{1}}{\partial t_{0} \partial t_{1}}-\frac{2 \delta \mu}{3} \frac{\partial^{4} \Psi_{2}}{\partial t_{0}{ }^{2} \partial \zeta_{0}{ }^{2}}-\frac{4 \delta \mu}{3} \frac{\partial^{4} \Psi_{1}}{\partial t_{0}{ }^{2} \partial \zeta_{0} \partial \zeta_{1}}- \\
& \frac{4 \delta \mu}{3} \frac{\partial^{4} \Psi_{1}}{\partial \zeta_{0}^{2} \partial t_{0} \partial t_{1}}
\end{aligned}
$$

Third-order, $O\left(\varepsilon^{3}\right)$,equation:

$$
\begin{aligned}
& \left(\frac{\partial \Psi_{1}}{\partial \zeta_{0}}\right)^{2} \frac{\partial^{2} \Psi_{1}}{\partial \zeta_{0}^{2}}+2 \frac{\partial \Psi_{1}}{\partial \zeta_{0}} \frac{\partial^{2} \Psi_{2}}{\partial \zeta_{0}^{2}}+2 \frac{\partial \Psi_{2}}{\partial \zeta_{0}} \frac{\partial^{2} \Psi_{1}}{\partial \zeta_{0}^{2}}+2 \frac{\partial \Psi_{1}}{\partial \zeta_{1}} \frac{\partial^{2} \Psi_{1}}{\partial \zeta_{0}{ }^{2}}+\frac{4}{3} \frac{\partial^{2} \Psi_{1}}{\partial \zeta_{0} \partial \zeta_{1}}+\frac{2}{3} \frac{\partial^{2} \Psi_{3}}{\partial \zeta_{0}{ }^{2}}+\frac{4}{3} \frac{\partial^{2} \Psi_{2}}{\partial \zeta_{0} \partial \zeta_{1}}+\frac{2}{3} \frac{\partial^{2} \Psi_{1}}{\partial \zeta_{1}{ }^{2}}+ \\
& 4 \frac{\partial \Psi_{1}}{\partial \zeta_{0}} \frac{\partial^{2} \Psi_{1}}{\partial \zeta_{0} \partial \zeta_{1}}=\frac{2 \delta}{3} \frac{\partial^{2} \Psi_{3}}{\partial t_{0}{ }^{2}}+\frac{4 \delta}{3} \frac{\partial^{2} \Psi_{2}}{\partial t_{0} \partial t_{1}}+\frac{4 \delta}{3} \frac{\partial^{2} \Psi_{1}}{\partial t_{0} \partial t_{2}}+\frac{2 \delta}{3} \frac{\partial^{2} \Psi_{1}}{\partial t_{1}{ }^{2}}-\frac{2 \delta \mu}{3} \frac{\partial^{4} \Psi_{3}}{\partial t_{0}{ }^{2} \partial \zeta_{0}{ }^{2}}-\frac{4 \delta \mu}{3} \frac{\partial^{4} \Psi_{2}}{\partial t_{0}{ }^{2} \partial \zeta_{0} \partial \zeta_{1}}- \\
& \quad \frac{4 \delta \mu}{3} \frac{\partial^{4} \Psi_{1}}{\partial t_{0}{ }^{2} \partial \zeta_{0} \partial \zeta_{2}}-\frac{4 \delta \mu}{3} \frac{\partial^{4} \Psi_{2}}{\partial \zeta_{0}^{2} \partial t_{0} \partial t_{1}}-\frac{8 \delta \mu}{3} \frac{\partial^{4} \Psi_{1}}{\partial t_{0} \partial t_{1} \partial \zeta_{0} \partial \zeta_{1}}-\frac{4 \delta \mu}{3} \frac{\partial^{4} \Psi_{1}}{\partial \zeta_{0}{ }^{2} \partial t_{0} \partial t_{2}}-\frac{2 \delta \mu}{3} \frac{\partial^{4} \Psi_{1}}{\partial t_{0}{ }^{2} \partial \zeta_{1}{ }^{2}}- \\
& \frac{2 \delta \mu}{3} \frac{\partial^{4} \Psi_{1}}{\partial t_{1}^{2} \partial \zeta_{0}^{2}}
\end{aligned}
$$

where $\psi_{1}$ is the function of fast as well as slow variables. 


\subsection{The solution of field equations:}

In the present section, an attempt should be made to acquire the solution of the field equations that govern different order terms in the perturbation expansion.

\subsubsection{The solution of $O(\varepsilon)$ order equation:}

The form of the differential equation presented in Eq. (31) indicates that we should look for the type of solution presented below:

$$
\psi_{1}=\varphi\left(\zeta_{1}, \zeta_{2}, \ldots ; t_{1}, t_{2}, \ldots\right) \exp \left[\mathrm{i}\left(\omega t_{0}-k \zeta_{0}\right)\right]+\text { c. c. }
$$

where $\omega$ denotes the angular frequency, $k$ denotes the wave number, $\varphi\left(\zeta_{1}, \zeta_{2}, \ldots ; t_{1}, t_{2}, \ldots\right)$ denotes the amplitude function depending on the slow variables, and c. c. denotes the complex conjugate of the equivalent quantity. As a result of introducing Eq. (34) into Eq. (31) and requiring the non-vanishing solution for $\varphi\left(\zeta_{1}, \zeta_{2}, \ldots ; t_{1}, t_{2}, \ldots\right)$, the following dispersion relation is obtained:

$$
\mathrm{D}(\omega, \mathrm{k})=\mathrm{k}^{2}-\delta \omega^{2}-\delta \mu \mathrm{k}^{2} \omega^{2}=0 .
$$

Here $\varphi\left(\zeta_{1}, \zeta_{2}, \ldots ; t_{1}, t_{2}, \ldots\right)$ stands for an unknown function, the governing equation of which will be acquired afterwards.

\subsubsection{The solution of $\mathrm{O}\left(\varepsilon^{2}\right)$, order equation:}

The form of Eq. (32) indicates that it is necessary to look for the type of solution for $\Psi_{2}$ presented below:

$$
\Psi_{2}=\sum_{\alpha=1}^{2} \Psi_{2}^{(\alpha)} e^{i \alpha \theta}+\text { c.c. }
$$

Here, the phasor $\theta$ is defined by $\theta=\omega t_{0}-k \zeta_{0}$ and $\Psi_{2}^{(1)}, \ldots, \Psi_{2}^{(-2)}$ are functions of slow variables of $\zeta_{0}$ and $t_{0}$. The equation for $\alpha=1$ mode presented below is acquired by introducing Eq. (34) and Eq. (36) into Eq. (32);

$$
\left[\mathrm{k}^{2}-\delta \omega^{2}\left(1+\mu \mathrm{k}^{2}\right)\right] \Psi_{2}^{(1)}+2 i k\left(1-\delta \mu \omega^{2}\right) \frac{\partial \varphi}{\partial \zeta_{1}}+2 i \delta \omega\left(1+\mu \mathrm{k}^{2}\right] \frac{\partial \varphi}{\partial t_{1}}=0
$$

Here, the coefficient of $\Psi_{2}^{(1)}$ is the dispersion relation and must be zero. The following is obtained by employing the dispersion relation: 


$$
2 i k\left(1-\delta \mu \omega^{2}\right) \frac{\partial \varphi}{\partial \zeta_{1}}+2 i \delta \omega\left(1+\mu \mathrm{k}^{2}\right] \frac{\partial \varphi}{\partial t_{1}}=0
$$

For obtaining non-zero solution for $\varphi$ that satisfies Eq. (38), it should have the form below:

$$
\varphi=\varphi\left(\xi, \zeta_{2}, \ldots ; t_{2}, \ldots\right) \quad, \quad \xi=\zeta_{1}-\lambda t_{1}
$$

Here, $\lambda$ stands for the group velocity of the wave, and it is described by:

$$
v_{g}=\lambda=\frac{k}{\omega \delta\left(1+\mu k^{2}\right)^{2}}
$$

Here, the function $\Psi_{2}^{(1)}$ represents another function, the governing equation of which is acquired from the higher-order expansion of the field quantities. The solution of Eq. (32) for $\alpha=2$ mode is obtained as follows:

$$
\Psi_{2}^{(2)}=\frac{3 i k^{3}}{\left[4 \mathrm{k}^{2}-4 \delta \omega^{2}-16 \delta \mu \mathrm{k}^{2} \omega^{2}\right]} \varphi^{2}=\frac{3 i k^{3}}{D(2 k, 2 \omega)} \varphi^{2}
$$

Here $D(l k, l \omega) \neq 0$ for $l=2,3, .$.

\subsubsection{The solution of $\mathrm{O}\left(\varepsilon^{3}\right)$, order equation:}

It is generally possible to express the solution for the order in question in terms of the phasor as follows:

$$
\Psi_{3}=\sum_{\alpha=1}^{3} \Psi_{3}^{(\alpha)} e^{i \alpha \theta}+\text { c.c. } \quad, \quad \theta=\omega t_{0}-k \zeta_{0}
$$

Only the first order equation in terms of the phasor is required for completing the solution for the unknown function $\varphi\left(\xi, \zeta_{2}, \ldots ; t_{2}, \ldots\right)$. By introducing Eqs. (34), (36) and (42) into Eq. (33), we obtain:

$$
\begin{gathered}
\frac{2}{3}\left[k^{2}-\delta \omega^{2}\left(1+\mu k^{2}\right)\right] \Psi_{3}^{(1)}+\frac{4 i \delta \omega}{3}\left[1+\mu k^{2}\right] \frac{\partial \Psi_{2}^{(1)}}{\partial t_{1}}+\frac{4 i k}{3}\left[1-\delta \mu \omega^{2}\right] \frac{\partial \Psi_{2}^{(1)}}{\partial \zeta_{1}}+ \\
\frac{4 i \delta \omega}{3}\left[1+\mu k^{2}\right] \frac{\partial \varphi}{\partial t_{2}}+\frac{4 i k}{3}\left[1-\delta \mu \omega^{2}\right] \frac{\partial \varphi}{\partial \zeta_{2}}+\frac{2}{3} \delta\left(1+\mu k^{2}\right) \frac{\partial^{2} \varphi}{\partial t_{1}{ }^{2}}-\frac{8}{3} \delta \mu k \omega \frac{\partial^{2} \varphi}{\partial t_{1} \partial \zeta_{1}}- \\
-\frac{2}{3}\left[1-\delta \mu \omega^{2}\right] \frac{\partial^{2} \varphi}{\partial \zeta_{1}{ }^{2}}-k^{4}|\varphi|^{2} \varphi+4 i k^{3} \Psi_{2}^{(2)} \varphi^{*}=0 .
\end{gathered}
$$

Here, the coefficient of $\Psi_{3}^{(1)}$ is the dispersion relation and must be zero. By rearranging Eq. (43), we have: 


$$
\begin{aligned}
\frac{4 \delta \omega}{3}\left[1+\mu k^{2}\right] \times & \left\{i\left(\frac{\partial \Psi_{2}^{(1)}}{\partial t_{1}}+\lambda \frac{\partial \Psi_{2}^{(1)}}{\partial \zeta_{1}}\right)+i\left(\frac{\partial \varphi}{\partial t_{2}}+\lambda \frac{\partial \varphi}{\partial \zeta_{2}}\right)\right\}-\frac{2}{3}\left[1-\delta \mu \omega^{2}-4 \delta \mu \lambda \mathrm{k} \omega-\delta \lambda^{2}(1+\right. \\
& \left.\left.\mu k^{2}\right)\right] \frac{\partial^{2} \varphi}{\partial \xi^{2}}-k^{4}|\varphi|^{2} \varphi+4 i k^{3} \Psi_{2}^{(2)} \varphi^{*}=0
\end{aligned}
$$

Here, the dependence of $\varphi$ on $\xi$ has been already utilized. Furthermore, in case of assuming that $\Psi_{2}^{(1)}$ depends on $t_{1}$ and $\zeta_{1}$ through $\xi$, then the drop occurs in the first terms in (44). As a result of presenting a new variable $\tau$ as $t_{2}=\tau, \zeta_{2}=\varepsilon \xi+\lambda \tau$, it is possible to read the second term in Eq. (44) as follows:

$$
\frac{\partial \varphi}{\partial t_{2}}+\lambda \frac{\partial \varphi}{\partial \zeta_{2}}=\frac{1}{\varepsilon} \frac{\partial \varphi}{\partial \xi}-\frac{1}{\varepsilon} \frac{\partial \varphi}{\partial \xi}+\frac{\partial \varphi}{\partial \tau}=\frac{\partial \varphi}{\partial \tau}
$$

By introducing the expression of $\Psi_{2}^{(2)}$ into (44), the nonlinear Schrödinger equation presented below is acquired:

$$
i \frac{\partial \varphi}{\partial \tau}+v_{1} \frac{\partial^{2} \varphi}{\partial \xi^{2}}+v_{2}|\varphi|^{2} \varphi=0
$$

where the coefficients $v_{1}$ and $v_{2}$ are defined by:

$$
\begin{gathered}
v_{1}=\frac{1}{2} \frac{\partial v_{g}}{\partial k}=-\frac{1}{2} \frac{\left[1-\delta \mu \omega^{2}-4 \delta \mu \lambda \mathrm{k} \omega-\delta \lambda^{2}\left(1+\mu k^{2}\right)\right]}{\delta \omega\left(1+\mu k^{2}\right)} \\
v_{2}=\frac{3}{4} \lambda k^{3}\left(1+\mu k^{2}\right)\left\{1-\frac{3 k^{2}}{\left[\mathrm{k}^{2}-\delta \omega^{2}-4 \delta \mu \mathrm{k}^{2} \omega^{2}\right]}\right\}
\end{gathered}
$$

The NLS equation appears in different fields as an equation that defines the self-modulation of one-dimensional monochromatic plane waves in dispersive media. The steady-state solution of the NLS equation, usually representing the wave trains that can be expressed in terms of Jacobian elliptic functions, contains bright and dark envelope solitons, a phase jump, and a plane wave with a constant amplitude as special cases. In order to determine how the presented initial data will develop in the long term for the asymptotic NLS equation in the form [40], the criterion of whether $v_{1} v_{2}>0$ or $v_{1} v_{2}<0$ is significant:

$$
\mathrm{A}(\xi, \tau)=\mathrm{V}(\eta) \exp [i(K \xi-\Omega \tau)], \quad \eta=\xi-v_{0} \tau, \quad v_{0}=\text { const }
$$

where $V(\eta)$ stands for a real function of $\eta$. In the mentioned situation, in case of $|\varphi|$ approaching a constant $V_{0}$ at infinity, the solution is presented by a non-linear plane wave:

$$
\varphi(\eta)=V_{0} \exp [i(K \xi-\Omega \tau)]
$$


where $\Omega=\sigma_{1} K^{2}-\sigma_{2} V_{0}{ }^{2}$. It is generally possible to acquire the solution in terms of the Jacobian elliptic functions by assuming that $v_{0}=2 \sigma_{1} K$. The specific functional form of the solution in question will be presented only for the limiting cases below. If there is an assumption that $V \rightarrow 0$ and $\frac{\partial V}{\partial \eta} \rightarrow 0$

as $|\eta| \rightarrow \infty$, for $\sigma_{1} \sigma_{2}>0$, the solution is presented by:

$$
\mathrm{V}(\eta)=A_{0} \operatorname{sech}\left[\left(\frac{v_{2}}{2 v_{1}}\right)^{1 / 2} A_{0} \eta\right]
$$

where $\Omega=\sigma_{1} K^{2}-\sigma_{2} A_{0}{ }^{2} / 2$. For $v_{1} v_{2}<0$, if $\varphi \rightarrow V_{0}$ and $\frac{\partial \varphi}{\partial \eta} \rightarrow 0$ as $|\eta| \rightarrow \infty$, the solution is given as follows:

$$
\mathrm{V}=V_{0} \tanh \left[\left(-\frac{v_{2}}{2 v_{1}}\right)^{1 / 2} V_{0} \eta\right]
$$

where $\Omega=\sigma_{1} K^{2}-\sigma_{2} V_{0}^{2}$. The mentioned solutions correlate to an envelope solitary wave and a phase jump, respectively. On the contrary, it is a well-known fact that the plane wave solution of the NLS equation is modulationally unstable in case of $v_{1} v_{2}>0$ or stable in case of $v_{1} v_{2}<0$.

\section{Numerical Results and Discussion}

This study examines nonlinear wave modulation in nanorods on the basis of nonlocal elasticity theory. In this study, numerical results are given for SWCNT with material and geometrical properties. In the light of the open literature, no consensus has been achieved on Poisson's ratio of nanotubes. The suggested values vary in a wide band $0.19 \sim 0.34$ [41]. Therefore, in the present study, $v$ is selected as 0.3 , and the nonlocal parameter $\mu$ is taken as $0 \sim 4 \times 10^{-18} \mathrm{~nm}^{2}$. Some material properties are taken as $\rho_{0}=2300 \mathrm{~kg} / \mathrm{m}^{3}, r_{0}=10^{-9} \mathrm{~m}, \mathrm{E}=1 \mathrm{TPa}$.

As mentioned previously, the features of both solutions of the NLS equation and the stability of the plane wave solution significantly depend on the sign of $v_{1} v_{2}$. Thus, giving more details on the change of $v_{1} v_{2}$ with the wave number will be interesting. The variation in $v_{1}$ for axial waves by wave number for three nonlocal parameter values are depicted in Fig 1 . As is seen from this figure for each selected values of $\mu, v_{1}$ values remain positive $\left(v_{1}>0\right)$. To clearly see the effect of $\mu$ on $v_{2}$, the graphs of $v_{2}$ by wave number are plotted in Fig 2 . This figure also shows that for each selected values of $\mu, v_{2}$ values remain positive $\left(v_{2}>0\right)$. The alterations of $v_{1} v_{2}$ for axial (longitudinal) waves and the wave number are presented in Fig 3 . As is seen from the mentioned figure, the plane wave solution for the wave in question is unstable for all values of the wave number for all nonlocal parameter values. 


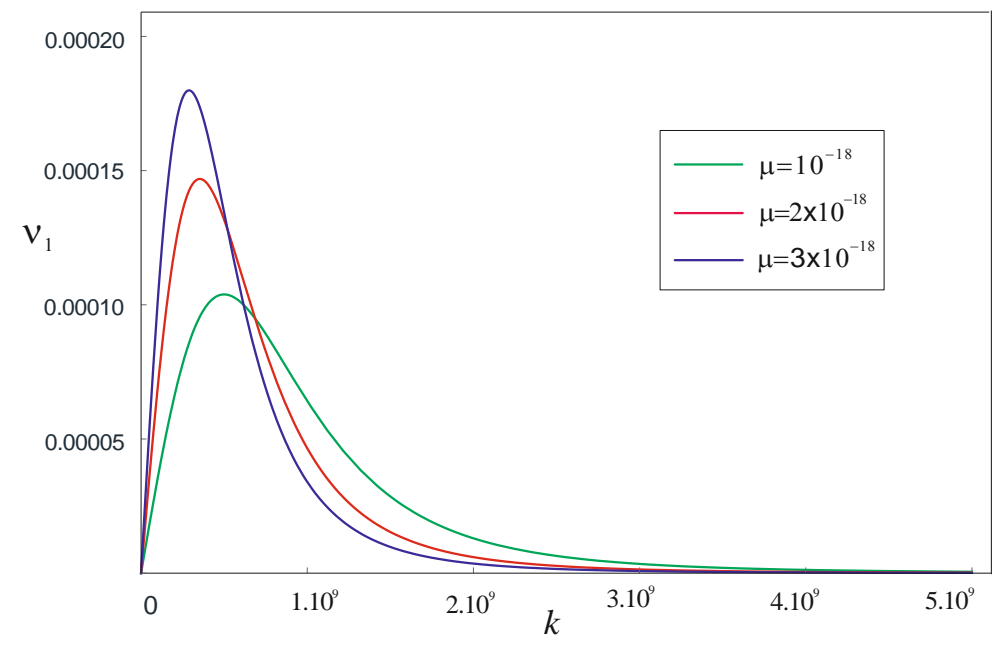

Fig. 1. Variation in the $v_{1}$ for axial waves by wave number for three nonlocal parameter values.

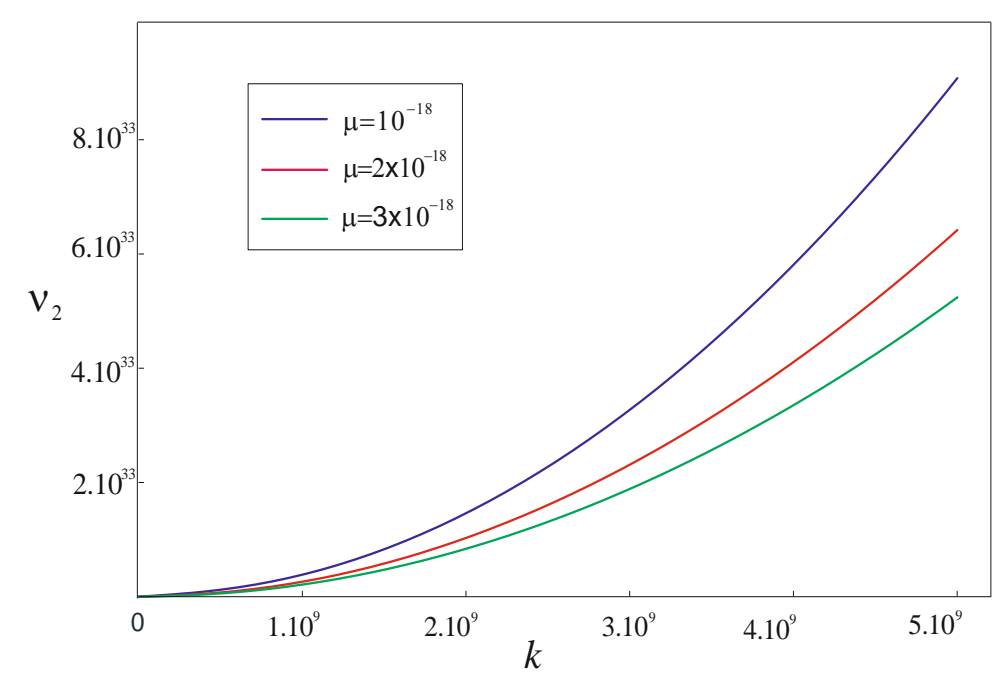

Fig. 2. Variation in the $v_{2}$ for axial waves by wave number for three nonlocal parameter values.

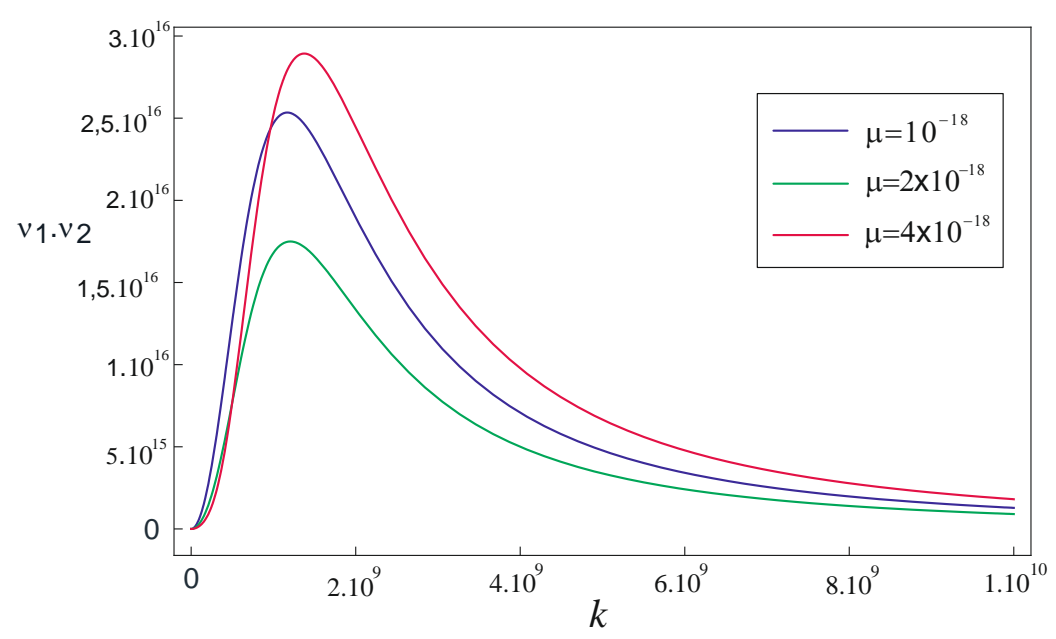

Fig. 3. Variation in the $v_{1} v_{2}$ for axial waves by wave number for three nonlocal parameter values. 
Regarding the alteration of the solution profile of the nonlinear Schrödinger equation, the splitstep Fourier method [42] was utilized, and the evolution equation was solved numerically. The change of the solution profile with variable $\eta$ at spatial time is presented in Fig. 4. Here, the nonlocal parameter was taken as $\mu=10^{-18}$. For $\mu=0$, there is no solitary wave profile because of non-dispersion.

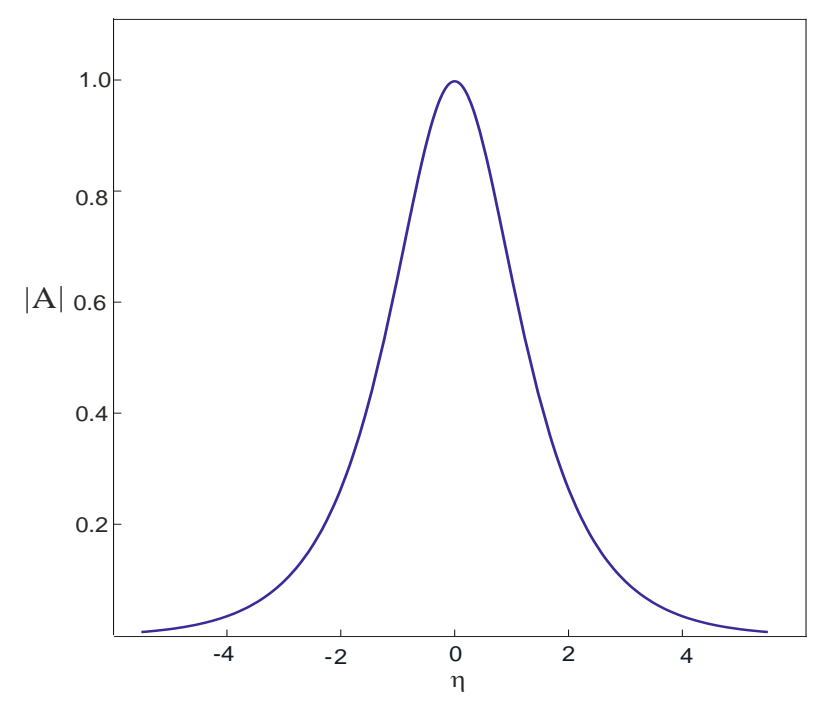

Fig. 4. Variation in the solution profile of the NLS equation by variable $\eta$ (at $\mu=10^{-18}$ )

The variation of wave frequency with the nonlocal parameter $\mu$ for various values of the wave number is given in Fig. 5. From the mentioned figure, it can be observed that a decrease in frequencies occurs with an increase in the nonlocal parameter. The frequency curves get closer to each other with an increase in nonlocal parameters.

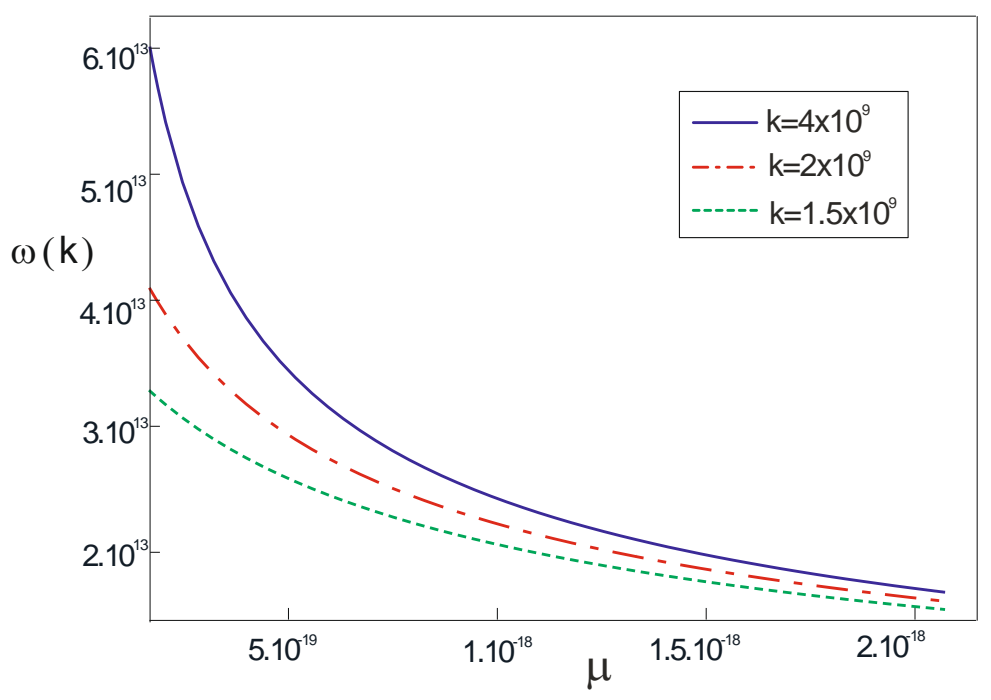

Fig. 5. Variation of wave frequency with the nonlocal parameter values for various values of the wave numbers 


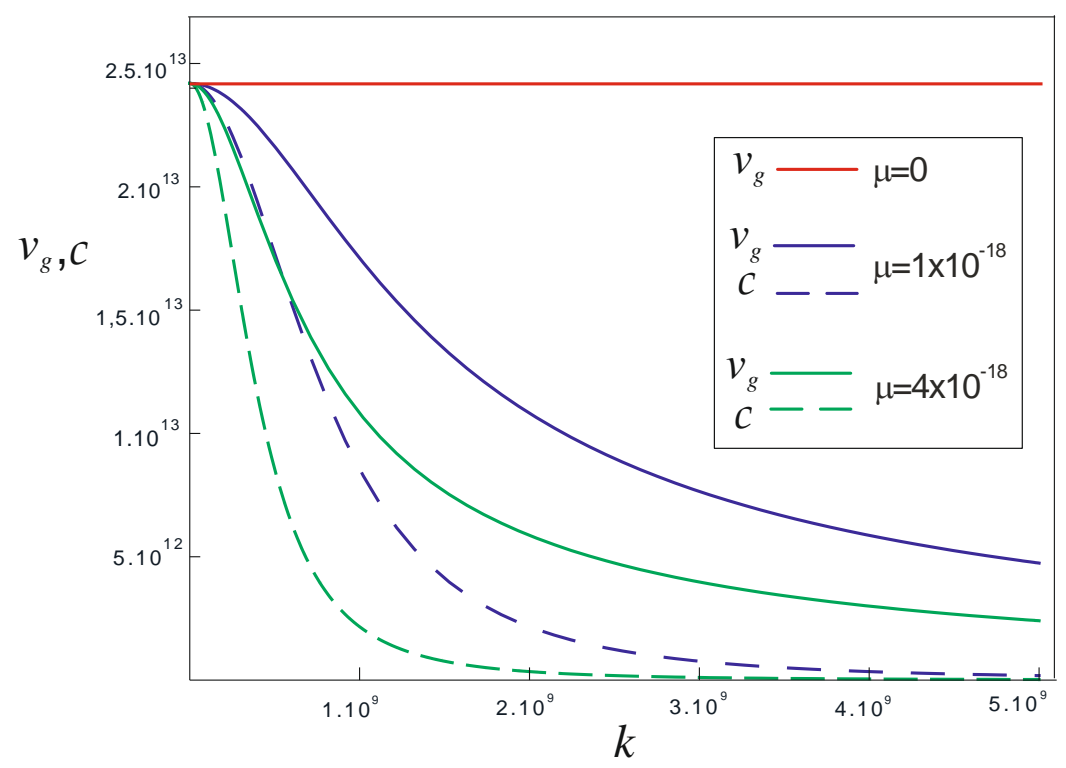

Fig. 6. Variation of phase and group velocities by the wave numbers for different nonlocal parameter values.

The impact of the nonlocal parameter on the velocities of nanotubes is plotted in Fig.6. From this figure, it can be observed that group and phase velocities decrease with increasing nonlocal parameters and wave numbers. For $\mu=0$, the group velocity is equal to phase velocity, which shows the non-dispersive situation.

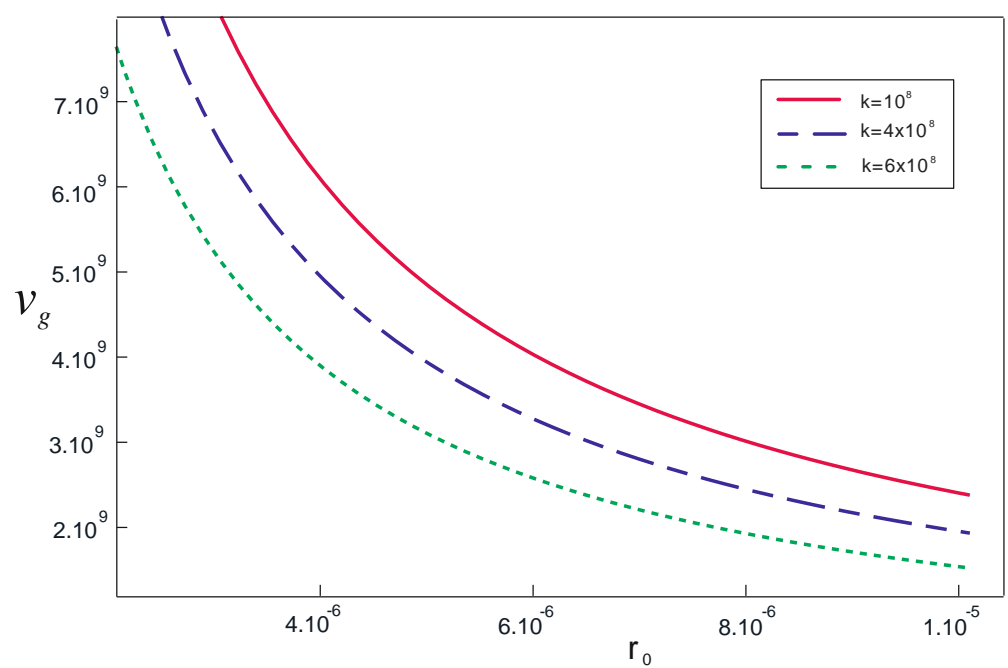

Fig. 7. Variation of group velocity with the radius for some different values of wave numbers.

The variation of group velocity with the radius for some different values of the wave number is presented in Fig.7. As is seen from the figure in question, with the increasing values of the radius, it is observed that group velocities decrease rapidly for different wave numbers. 


\section{Conclusions}

In the present study, the self-modulation of weakly nonlinear and strongly dispersive waves in nanorods was examined, and the nonlinear Schrödinger (NLS) equation was obtained as an evolution equation of a slowly changing amplitude of the carrier wave. Solutions for the NLS equation for nonlinear plane waves, envelope solitary waves, and phase jump solutions are also provided. In addition to these, in the present study, due to nonlocality, the elastic medium has a dispersive character. The nonlocal parameter $\mu$ represents the dispersive character of the medium. If $\mu=0$, this leads to the nonlinear motion of classical elasticity. In the wave propagation analysis, together a linear and local situation represents a non-dispersive character. A linear and non-local situation represents a dispersive character. Besides, the nonlinear and local situation represents a non-dispersive character. However, in case of a non-linear and nonlocal situation, nonlinearity and dispersion balance with each other and a solitary wave profile arise.

In numerical calculations, it is shown that the plane wave solution of the NLS equation is modulationally unstable in case of $v_{1} v_{2}>0$ for all values of the wave number for all nonlocal parameter values. This situation corresponds to the envelope solitary wave solution. For $\mu=0$, there is no solitary wave profile because of a non-dispersion. Wave frequency curves and group velocity curves are plotted with the wave number, and it is shown that nonlocal frequency and velocity curves are approximately the same with the literature [24,43]. To see the small-scale effect of nanorods, the variation of wave frequencies was examined, and group velocities changed with the radius of a nanorod. It is shown that frequencies and group velocities are decreasing with the increasing nanorod radius. It is observed that the nonlocal parameter has an obvious effect on wave frequencies and velocities. It is expected that the nonlinear modulation introduced herein will be useful for studies on the nanostructure.

\section{References}

[1] Eringen, A. C., Suhubi, E. S., Nonlinear theory of simple micro-elastic solids-I, International Journal of Engineering Science, 2, 189-203, 1964.

[2] Eringen, A. C., Simple microfluids, International Journal of Engineering Science, 2, 205$217,1964$.

[3] Eringen, A. C., Theory of micropolar elastisity in Fracture (Edited by H. Liebowitz), Vol. II Academic Press, New York, 1968.

[4] Kafadar, C. B., Eringen A. C., Micropolar Media-I. The classical theory, International Journal of Engineering Science, 9, 271-305, 1971.

[5] Eringen, A. C., Nonlocal polar elastic continua, International Journal of Engineering Science, 10, 1-16, 1972.

[6] Demiray, H., A nonlocal continuum theory for diatomic elastic solids, Int. J. Eng. Sci., 15, 623-644, 1977

[7] Eringen, A. C., On differential equations of nonlocal elasticity and solutions of screw dislocation and surface waves, Journal of Applied Physics, 54, 4703-4710, 1983. 
[8] Toupin, R. A., Elastic materials with coupled stresses, Archive for Rational Mechanics and Analysis, 11, 385, 1962.

[9] Park, S. K., Gao, X. L., Bernoulli-Euler beam model based on a modified coupled stress theory, Journal of Micromechanics and Microengineering, 16 (11),23055-2359, 2006.

[10] Ma, H. M., Gao, X. L., Reddy J. N., A microstructure-dependent Timoshenko beam model based on a modified couple stress theory, Journal of the Mechanics and Physics of Solids, 56(12), 3379-3391, 2008.

[11] Murmu, T., Pradhan, S. C., Small-scale effect on the vibration on the nonuniform nanocantiliver based on nonlocal elasticity theory, Physica E, 41, 1451-1456, 2009.

[12] Senthilkumar, V., Pradhan, S. C., Pratap, G., Small-scale effect on buckling analysis of carbon nanotube with Timoshenko theory by using differential transform method, $A d v$. Sci. Lett., 3, 1-7, 2010.

[13] Rahmani, O., Pedram, O., Analysis and modelling the size effect on vibration of functionally graded nanobeams based on nonlocal Timoshenko beam theory, International Journal of Engineering Science, 77, 55-70, 2014.

[14] Eringen, A. C., Linear theory of nonlocal elasticity and dispersion of plane waves, International Journal of Engineering Science, 10, 1-16, 1972.

[15] Eringen, A. C., Edelen, D. G. B., On nonlocal elasticity, International Journal of Engineering Science, 10, 233-248, 1972.

[16] Thai, H. T., A nonlocal beam theory for bending, buckling and vibration of nanobeams, International Journal of Engineering Science, 52, 56-64, 2012.

[17] Aydogdu, M., Axial vibration of the nanorods with the nonlocal continuum rod model, Physica E: Low-dimensional Systems and Nanostructures, 41(5), 861-864, 2009.

[18] Aydogdu, M., Axial vibration analysis of nanorods (carbon nanotubes) embedded in an elastic medium using nonlocal theory, Mechanics Research Communications, 43, 34-40, 2012.

[19] Lim, C. W. and Yang, Y., Wave propagation in carbon nanotubes: nonlocal elasticityinduced stiffness and velocity enhancement effects, J. Mech. Mater. Struct., 5, 459-476, 2010 .

[20] Hu, Y. G., Liew, K. M., Wang, Q., He, X. Q., Yakobson, B. I., Nonlocal shell model for elastic wave propagation single- and double-walled carbon nanotubes, J. Mech. Phys. Solids, 56: 3475-3485, 2008.

[21] Wang, Q., Varadan, V. K., Wave characteristics of carbon nanotubes, Int. J. Solids Struct., 43, 254-265, 2006.

[22] Narendar, S., Gopalakrishnan, S., Temperature effects on wave propagation in nanoplates, Compos. Part B, 43, 1275-1281, 2012. 
[23] Narendar, S., Gopalakrishnan, S., Nonlocal scale effects on wave propagation in multiwalled carbon nanotubes, Comput. Mater. Sci., 47, 526-538, 2009.

[24] Aydogdu, M., Longitudinal wave propagation in nanorods using a general nonlocal unimodal rod theory and calibration of nonlocal parameter with lattice dynamics, Int. J. Eng. Sci., 56, 17-28,2012.

[25] Aydogdu, M., Longitudinal wave propagation in multiwalled carbon nanotubes, Composite Structures, 107: 578-584, 2014.

[26] Wu, X. F., Dzenis, Y. A. ,Wave propagation in nanofibers, J. App. Phys., 100, 124318, 2006.

[27] Challamel, N., Rakotomanana, L., Marrec, L. L., A dispersive wave equation using nonlocal elasticity, Comptes Rendus Mecanique, 337, 591-595, 2009.

[28] Narendar, S., Gopalakrishnan, S., Nonlocal scale effects on wave propagation in multiwalled carbon nanotubes, Comput. Mater. Sci., 47, 526-538, 2009.

[29] Narendar, S., Terahertz wave propagation in uniform nanorods: a nonlocal continuum mechanics formulation including the effect of lateral inertia, Physica E: Low-dimensional Syst. Nanostruct., 43, 1015-1020, 2011.

[30] Erbay, S., Erbay, H. A., Dost, S., Nonlinear wave modulation in micropolar elastic mediaI. Longitudional waves, International Journal of Engineering Science, 29 (7), 845-858, 1991.

[31] Erbay, H. A., Erbay, S., Nonlinear wave modulation in fluid filled distensible tubes, Acta Mechanica, 104, 201-214, 1994.

[32] Akgun, G., Demiray, H., Nonlinear wave modulation in a pre-stressed viscoelastic thin tube filled with an inviscid fluid, Int. J. Non-linear Mech., 34, 571-588, 1999.

[33] Akgun, G., Demiray, H., Modulation of non-linear axial and transverse waves in a fluidfilled thin elastic tube, Int. J. Non-linear Mech., 35, 597-611, 2000.

[34] Erbay, H. A., Erbay, S., Erkip, A., Unidirectional wave motion in nonlocally and nonlinearly elastic medium: the $\mathrm{KdV}$, BBM and $\mathrm{CH}$ equations, Nonlinear Waves, 64, 256$264,2015$.

[35] Duruk, N., Erbay, H. A., Erkip, A., Blow-up and global existence for a general class of nonlocal nonlinear coupled wave equations, J. Differ. Equations, 250, 1448-1459, 2011.

[36] Malvern, L. E., Introduction to the Mechanics of a Continuum Medium, Prentice Hall, Englwood Cliffs, New Jersey, 1969.

[37] Mousavi, S. M., Fariborz, S. J., Free vibration of a rod undergoing finite strain, J. of Physics Conferans Series, 382(1), 2012.

[38] Fernandes, R., El-Borgi, S., Mousavi, S. M., Reddy, J.N., Mechmoum, A., Nonlinear sizedependent longitudinal vibration of carbon nanotubes embedded in an elastic medium, Phisica E, 88, 18-25, 2017. 
[39] Jeffrey, A., Kawahara, T., Asymptotic Methods in Nonlinear Wave Theory, Pitman, Boston, 1982.

[40] Lamb Jr., G. L., Mc Laughlin, D. W., in: Bullough, R. K., Coudrey, P. J. (Eds), Aspect of Soliton Physics:in Solitons, Springer, Berlin, 1980.

[41] Tu, Z-C., Single walled and multiwalled carbon nanotubes viewed as elastic tubes with the effective Young's moduli dependent on layer number, Physics Rev. B, 65, 233-237, 2002

[42] Taha, T. R., Ablowitz, M. J., Analytical and numerical aspects of certain nonlinear evolution equations. II Numerical nonlinear Schrödinger equation, J. Comput. Phys., 55, 203-230, 1984

[43] Wang, H., Dong, K., Men, F., Yan, Y. J., Wang, X., Influences of longitudinal magnetic field on wave propagation in carbon nanotubes embedded in elastic matrix, Appl. Math. Model., 34, 878-889, 2010. 